\title{
Pegasis Double Cluster Head Hybrid Congestion Control in Wireless Sensor Networks
}

\author{
Abdul Ali, and M. Vadivel
}

\begin{abstract}
Wireless sensor network (WSN) is the fastest growing technology that dominates the future world into wireless communication. It is a collection of a number of self-governing sensor nodes responsible to sense, process and manipulate the nodes. The sensor nodes are regulated by a battery where the network gets failed if the battery is dead. Thus, energy is an important factor to be efficiently used. Furthermore, congestion occurs in WSN when the incoming traffic load exceeds the capacity of the network. The major factors that lead to congestion are buffer overflow, varying rates of transmission, packet collision, and many-to-one data transmission. Due to these, the network suffers from packet loss, queuing delay, end-to-end delay, decrease in network lifetime, and increase in energy consumption. Hence, a clustering-based routing protocol is introduced in this paper to improve the performance of the network and reduce congestion. In the proposed method, Power-Efficient Gathering in Sensor Information Systems (PEGASIS) double cluster head with artificial neural network (ANN) is utilized to analyze the overall network lifetime. The proposed technique is comprised of four phases: clustering the network nodes, cluster head $(\mathrm{CH})$ selection, chain formation, and secondary $\mathrm{CH}(\mathrm{SCH})$ selection. The sensor nodes are initially clustered with the firefly algorithm in which the cluster heads of each node are elected via an artificial neural network. Meanwhile, chain formation is processed by PEGASIS double cluster head (PDCH) and the SCH is selected through grey wolf optimizer (GWO) to afford equivalent energy utilization between the sensor nodes. The simulation outcomes proved that the proposed method efficiently increases the lifetime of the network and reduces congestion level in WSN.
\end{abstract}

Index Terms-Wireless sensor networks, cluster, routing, energy consumption, firefly algorithm, PEGASIS double cluster head, artificial neural network, grey wolf optimizer.

\section{INTRODUCTION}

$\mathbf{T}$ HE wireless sensor network is a combination of networks containing small, low cost, energy constrained and unreliable multifunctional micro-sensor nodes [1]. The information captured on every sensor node is transferred to the base station (BS). In general, the outstanding performance of WSNs includes fault tolerance, rapid deployment, self-establishing, timely response, etc. Therefore, WSN is widely used in several ranges of applications including military purposes, surveillance in industrial product, smart homes and health care

Manuscript received February 10, 2021; revised April 23, 2021. Date of publication July 12, 2021. Date of current version July 12, 2021. The associate editor prof. Miroslav Joler has been coordinating the review of this manuscript and approved it for publication.

A. Abdul is Research Scholar in the Department of Computer Science and Engineering, Sathyabama Institute of Science and Technology, Chennai, India (e-mail: abdulalikm86@gmail.com).

M. Vadivel is a Professor in the Department of Electronics and Communication Engineering, Vidya Jyothi Instituite of Technology, Hyderabad, India.(email:drmvadivel79@gmail.com).

Digital Object Identifier (DOI): 10.24138/jcomss-2021-0032
[2] [3]. Even though WSNs provide amazing performance, it has its own limitations in the implementation of security constrained resources in memory, computing, battery life, and bandwidth [4]. Thus, the sensed and transmission signals should be carefully maintained and used. The performance of WSNs is improved with the implementation and careful maintenance of routing. It is employed for transmitting the information from sensor nodes to the base station thereby developing communication between them. The protocols in routing are classified based upon the node involvement, functioning of the protocol, and network architecture. The major drawbacks of routing include reduction in a network lifetime, increase in energy consumption among the nodes, node deployment, scalability and connectivity issues, coverage, and security problems [6]. Hence, various protocols have been developed to enhance the performance of routing. Besides, an alternative method for increasing the network lifetime is clustering.

Clustering is a protocol that gathers nodes and improves the efficiency of network. The nodes (hubs) in the network capture related interior variables from nature and transmit them to the particular cluster head. Finally, the sensed data are gathered by the CHs and send to the BS [7]. Clustering based networks are used in WSN because of the following reasons. (i) Clustering performs data gathering at $\mathrm{CH}$ level and minimizes energy consumption by removing the unnecessary information. (ii) During routing, the selected $\mathrm{CHs}$ only have to perform the action of route between other cluster heads with a bit of route information. Moreover, it will enhance the network scalability. (iii) Clustering keeps up the communication data transfer capacity since sensor hubs interact only with their corresponding $\mathrm{CHs}$ and hence avoids unnecessary data transmission. (iv) Clustering can propose the optimized management scheme. The CHs in clustering techniques receive more data from both cluster members and other $\mathrm{CH}$ s to exchange information to the sink node [8]. In cluster-based routing protocols, sensor nodes (SNs) get split into more clusters to limit the energy utilization for significant distance communication [15]. Hence, clustering minimizes energy utilization by balancing the energy in nodes through energy reduction among CHs and different hubs [10].

If the packets moved to the destination in WSN go beyond the storage capacity, then packet drop occurs. This leads to the retransmission of dropped packets in the network causing overhead network traffic and congestion. The traffic congestion in WSNs reduces the whole network efficiency because of data loss, reduced quality of service, and delay in data transmission. To overcome these difficulties, a novel routing protocol is 
proposed in this paper. The sensor node is initially clustered to examine a group of unlabeled information that minimizes the power consumption and interaction overhead. Furthermore, the cluster-based techniques utilize sensor nodes as cluster heads to communicate effectively for gathering data as well as consuming energy in the network. The $\mathrm{CH}$ s communicate with cluster nodes to accumulate or shrink data before transferring to the sink node [12]. Currently, artificial intelligence has been deeply inspected for the improvement of energy efficiency in WSN. The sensor nodes are gathered to form a chain structure for the transmission of packets to the base station. If the nodes are dead during transmission, then the chain has to rebuild to bypass the dead node. Therefore, a $\mathrm{CH}$ takes responsibility to transmit data to the sink node and enhances energy efficiency. The node stands alive if the energy of the hub is greater than the threshold value. This helps to improve the lifespan of the network and control congestion.

The main contribution of this paper is described as follows. The sensor nodes are usually grouped in the form of clusters for efficient data communication. In the proposed methodology, clustering is done through firefly algorithm inspired by the flashing behavior of fireflies. The networks suffer from congestion because of continuous data transmission to the BS. Therefore, a $\mathrm{CH}$ is appointed via ANN for each cluster to interact with the central base station. A set of nodes possessing energy-rich content are selected as $\mathrm{CHs}$ from the deployed sensor nodes. The nodes in WSNs are interconnected in the form of a chain via the PEGASIS-DCH algorithm to maintain effective power utilization throughout the network lifetime. Additionally, it allows data transmission and reception from adjacent nodes to the sink node. For this purpose, an $\mathrm{SCH}$ is nominated via GWO among the chain nodes to perform this action. Finally, the proposed model is experimentally evaluated and compared with existing works. From simulation results, it can be proved that the proposed method is better than previous approaches.

The rest of the paper is summarized as follows. Section II describes a literature review on recently developed techniques. Preliminaries of the implemented method are elaborated in Section III. In Section IV, the proposed technique concerning energy efficiency is presented. Experimental results with simulation are explained in Section V. Conclusion and future work are mentioned in Section VI.

\section{LiterATURE REVIEW}

In WSNs, clustering-based techniques are the ideal processes used for the valuable depletion of energy among sensor nodes. Srivastava et al. [13] implemented a rate-based congestion control algorithm based on clustered routing. The primary focus of this method is to prevent congestion and reduce end-to-end delay with a reduced packet failure rate. The network operates in a rate control process to decrease the endto-end delay and attain better performance for large-time set up experiments. The cluster formation of the nodes is conducted with K-means and Greedy algorithm. The rate control task is carried out using firefly protocol, highly recommended for packet transmission with a high delivery ratio. The packets are transferred through an ant colony optimization-based routing protocol to get increased throughput. Sharma et al. [15] suggested a new Gauss-sigmoid cluster routing protocol that significantly improved network lifetime and network throughput when compared to conventional clustering algorithms. Besides, the low energy adaptive clustering hierarchy (LEACH) protocol undergoes an issue in the formation of the number of clusters. Hence, Kirsan et al. [16] proposed a new LEACH focused on the selection of the best cluster head nodes. In this approach, the cluster heads are selected by the base stations that point out the higher energy clusters. The new LEACH could minimize the energy utilization of each sensor node with an improvement in the network lifetime. However, the new LEACH is found efficient to select only a few $\mathrm{CH}$ sensor nodes.

Gupta et al. [17] proposed an optimized hybrid energyefficient distributed (OHEED) protocol for heterogeneous WSN on different levels of node heterogeneity (i.e., 1-level, 2-level, 3-level, and multi-level) named as heterogeneous OHEED protocols. It can handle efficiently both homogeneous and heterogeneous nodes in the network. It represents that improving the node level by heterogeneity and stability of the Hetero-OHEED algorithm improves the network lifetime. Thus, it searches only the high residual energy sensor nodes during CH selection. Similarly, Sackey et al. [18] invented an energy-efficient clustering-brain storm optimization (EECBSO) approach for increasing the energy efficiency in WSNs. BSO method is used for best cluster head selection that strengthens the energy efficiency, packet data rate, and coverage percentage. The $\mathrm{CHs}$ may drain its energy after several transmissions during $\mathrm{CH}$ selection. To overcome this issue, Tomar et al. [19] illustrated an energy-efficient gravitational search algorithm (GSA) and fuzzy-based clustering with hop count routing. A supercluster head is elected by a fuzzy inference system among the preferred CHs. Furthermore, data security is monitored during data transmission. Hamzah, et al. [20] established a fuzzy logic model for $\mathrm{CH}$ selection. This approach attains the best possible outcome of energyefficient routing in WSNs. However, the use of the fuzzy logicbased energy-efficient method in WSNs reduced the distance between the executions of CHs. Gini index is also adopted to estimate the clustering protocols and energy proficiency capacity to adjust the conveyance of energy in sensor nodes. This improves energy productivity as far as network lifetime, energy utilization adjustments among sensor hubs for various network sizes and geographies. Though, it improves only an average rate of first and half node dead. As a result, neural networks have been recommended for further development.

Shoubin et al. introduced a data fusion algorithm based on a neural network to enhance the data fusion efficiency and prolong the network lifetime. This algorithm interacts with the backpropagation neural network to enhance the routing protocol of WSN. In this manner, it diminishes the information measure for transmission, saves energy efficiency, and improves the network lifetime. Nevertheless, there occurs network congestion in packet arrival to the base station. Wang et al. [22] introduced a chain-based routing protocol called enhanced PEGASIS (EPEGASIS) to minimize the energy 
consumption through a short average transmission distance between sensor nodes and mobile sink. All the sensor nodes in EPEGASIS utilize an optimal interaction gap to find the relay node from its adjacent node for data transfer. On the other hand, specific nodes in particular locations make use of higher energy in addition to hotspot issues that is not fully resolved. Therefore, meta-heuristic optimization algorithms are introduced in PEGASIS methodologies. Tao et al. [23] suggested PEGASIS routing protocol with ant colony optimization and neural network termed as ACON-PEG. It constructs a chain along with the supply of energy as a main key factor. When related to traditional PEGASIS, the ACON-PEG can accomplish global development. The chain formation is processed to make the path even better where the transmission distance of data packets becomes much less. The application of ant colony optimization selects a perfectly possible way to transmit data to the sink node [15]- [23]. Though it forms a long chain for data transmission, the PDCH method is adopted in this article to reduce the delay time.

Motivation. In WSNs, congestion is occurred due to the nature of the wireless channel, channel interference, reporting rate, slow processor, limited memory, and limited energy in nodes. Hence, protocols designed for WSNs must be lightweight and scalable to extend the lifetime of the network to the maximum extent. When the node transmits packets more than the receiver capacity, there will be a loss of packets leading to packet re-transmission, network traffic, and congestion [24]. Hence, we discussed the firefly clusteringbased routing protocol in our paper to improve the energy efficiency of the nodes and reduce the congestion level. It also develops an interactive relationship between nodes to enhance the bandwidth of network consumption. The advantage of using clustered networks is effective for resource allocation and reuse of occupied bandwidth. Subsequently, the chain formation is formed by the PEGASIS-PDCH algorithm. Moreover, the cluster head node is estimated via ANN and the secondary $\mathrm{CH}$ node is selected via the GWO algorithm. The proposed method is aimed to increase the energy efficiency and control congestion in WSN.

\section{PRELIMINARIES}

The proposed method uses PEGASIS-PDCH with ANN to enhance the network lifetime and increase the energy efficiency between nodes. The network undergoes four phases like clustering the sensor nodes by firefly algorithm, cluster head selection through ANN, chain formation with PEGASIS double cluster head selection and $\mathrm{SCH}$ selection via GWO algorithm. The block diagram of the proposed method is shown in Figure 1. The preliminaries behind the proposed concept are discussed in this section.

\section{A. Network Model}

The network design is homogeneous in the proposed WSN. It contains a sink node with more sensor nodes having equivalent capacity of battery power, processing power, and storage. Consider a two-network model with sink nodes located inside the network in one model like Figure 2 (a) and cluster heads

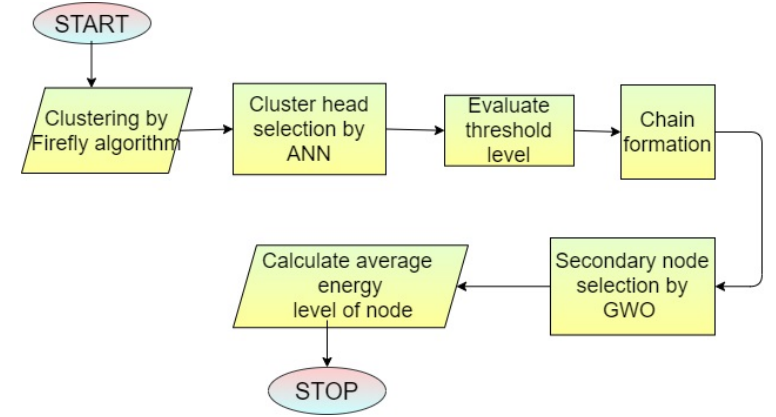

Fig. 1. Block diagram for the proposed method.

in the other network model like Figure 2 (b). The nodes in a specific cluster are assembled based on the minimum separation from the $\mathrm{CH}$.

\section{B. Energy Model}

Energy is a significant factor in WSNs, but sensor nodes usually have restricted energy. In spite, the batteries used in WSNs cannot be reused because once the battery is drained there is no other way of recharging again. The transceiver unit utilizes more energy while transferring information to the base station. The energy consumption model is shown in Figure 3. The transmission energy consumption is $E_{T X}$ to transmit packets at a distance $d$. The overall energy consumption of each node to transmit $p$-bit of data to propagation distance $q$ is calculated by Equation (1).

$$
E_{T X}(p, q)=\left\{\begin{array}{l}
E_{e l e} * p+E_{s t} * p+d^{2} \quad d<d_{0} \\
E_{e \mid e} * p+E_{m n} * p+d^{4} \quad d \geq d_{0}
\end{array}\right.
$$

$E_{e \mid e}$ shows the energy utilized by the transmitter or receiver circuitry. $E_{s t}$ is the energy used by the transmitter amplifier for the free space model and $E_{m n}$ denotes the energy consumed by the transmitter amplifier in the network. The energy consumed on receiving the packets is determined as in Equation (2) where $E_{R} X$ denotes energy consumed on receiving the data. Moreover, $d_{0}$ is the threshold transmission distance expressed in Equation (3).

$$
\begin{gathered}
E_{R X}(p, q)=E_{\text {ele }} * p \\
d_{0}=\sqrt{\frac{E_{s t}}{E_{m n}}}
\end{gathered}
$$

\section{Firefly Algorithm}

Firefly algorithm (FA) is a nature-stimulated method of the flashing conduct of the firefly [25]. The primary aim of this algorithm is to discover a feasible solution for deciding the best fitness function [26]. It follows three principles as discussed

- Fireflies are attracted disrespect to gender with the goal that they are pulled in to one another.

- The attractiveness of the firefly is calculated by the brightness value. So, fewer less-bright fireflies are moved 


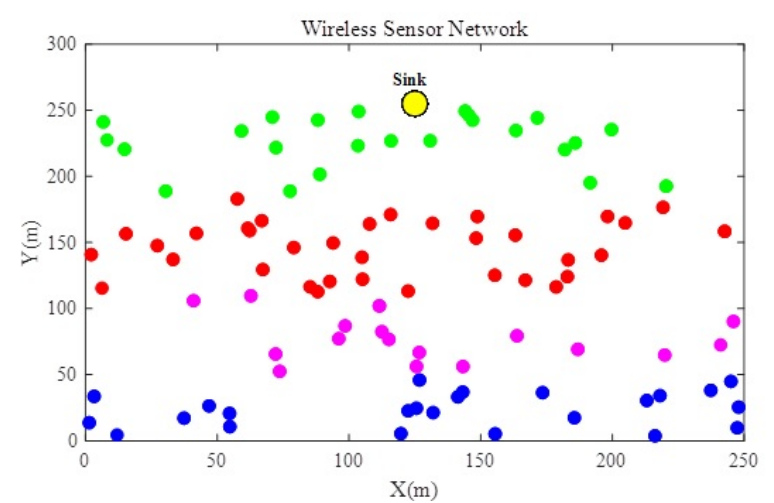

(a)

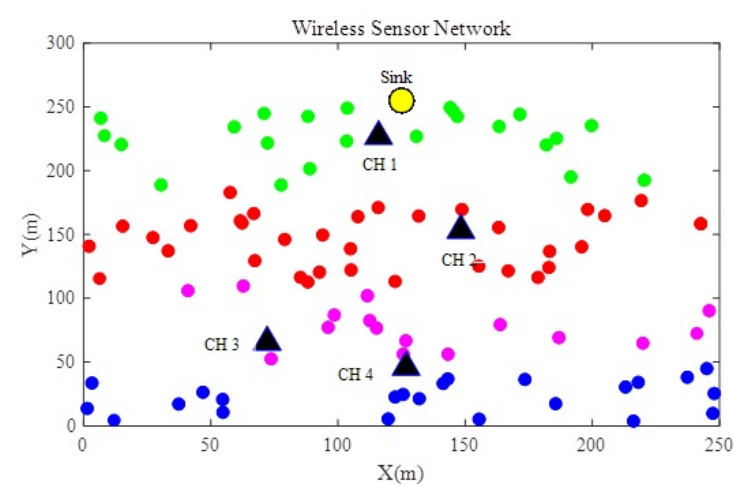

(b)

Fig. 2. Network model: (a) Sink inside network without cluster head (b) with cluster head.

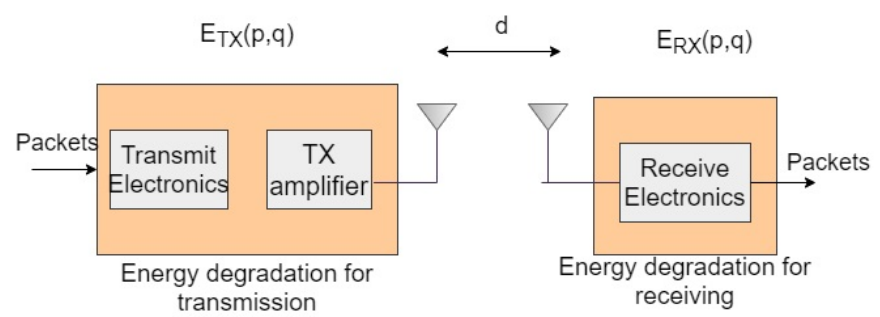

Fig. 3. Energy consumption model.

towards the brighter fireflies. Attractiveness is reduced by expanding the distance between them. When there is no brighter firefly, they move haphazardly.

- The brightness of the firefly is dictated via objective function area.

\section{Artificial Neural Network}

ANN is a computational three-layer network model that simulates the biological nerve cells or neurons in the brain. It is a middle region-based effective registering network that acquires knowledge from logical neural networks. [27]. The neurons in ANN establish a communication link to create a connection with other neurons as shown in Figure 4. The input signal or data is interconnected with weights in the communication link.

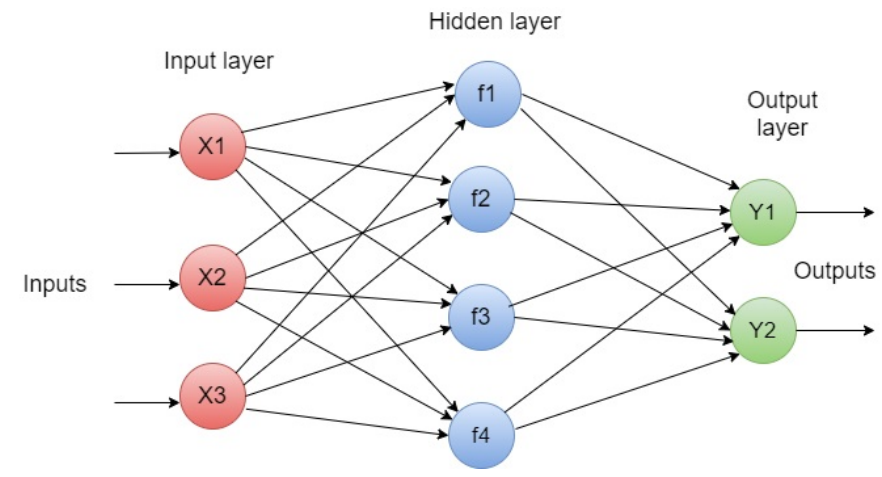

Fig. 4. Model of an artificial neural network.

\section{E. PEGASIS- Double Cluster Head}

PDCH uses dual CHs namely the primary cluster head (PCH) and secondary cluster head (SCH) to develop communication in a single chain. $\mathrm{PCH}$ is selected from the main chain and $\mathrm{SCH}$ is selected from the branch chain [28]. The functions of the primary head include gathering and combining data from all the nodes in the cluster. The cluster head identifies unnecessary data and removes it. Then the primary cluster head transfers this data to the secondary cluster head. The functions of $\mathrm{SCH}$ include collecting all the data from the primary cluster head and transmitting it to the base station. It performs aggregation before transmitting the data in which a few data is removed and some energy is consumed.

\section{F. Grey Wolf Optimizer}

GWO is a meta-heuristic algorithm used as a solution for optimization issues. It is motivated by the natural existent hierarchy of hunting mechanisms of a pack of grey wolves [29]. The grey wolves usually attack the prey by forming a group with a range of 5-12 wolves. These wolves are further divided into four categories based on their characteristics as alpha, beta, delta, and omega in a hierarchical manner [30]. The alpha wolf is powerful among these wolves and it leads the team. The second powerful wolf is the beta wolf that takes charge and leads the entire pack of wolves in the absence of the alpha wolf. The third and fourth are respectively the delta and omega wolves commonly called minimum active wolves [31]. The GWO mechanism is adopted in this paper for the selection of secondary $\mathrm{CHs}$.

\section{Proposed Energy Balancing Cluster-Based ROUTING PROTOCOL}

The proposed method is represented in Figure 5. The WSN consists of several sensor nodes in which the initial clustering process is done with the firefly algorithm. The cluster group selects a $\mathrm{CH}$ as a head member of the group via ANN. The condition is checked whether the energy of $\mathrm{CHs}$ is less than the threshold or not. If it is less, the $\mathrm{CH}$ selection process is carried out, otherwise the subsequent process is performed. The nodes form a chain for transferring data to the BS through 
the PDCH method. The formation of two cluster heads in the proposed network improves energy efficiency. Moreover, the secondary $\mathrm{CH}$ in PDCH is selected using the GWO protocol. If the energy in nodes is greater than the threshold, the node is alive otherwise it is considered as a dead node. Finally, the network updates the status of alive nodes and move to the next round of selection. Once the node is dead, no more rounds will present. Finally, the energy utilized by the network is evaluated for all rounds. The proposed method undergoes four phases like clustering, cluster head selection, chain formation, and secondary $\mathrm{CH}$ selection.

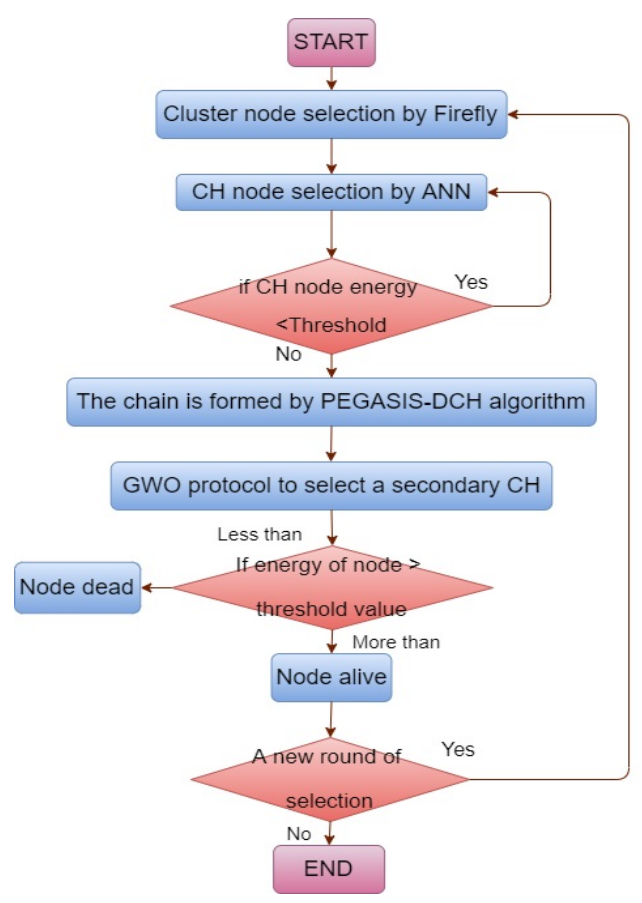

Fig. 5. Flow chart of the proposed PDCH-ANN algorithm.

1) Clustering: Initially, the indefinite nodes are identified as auxiliary cluster heads. The sensor node distant from the base station is considered as the assistant head node and the nearest nodes are its member. When the first cluster is formed, the farthest nodes from the un-clustered nodes are chosen as subsequent assistant group heads. This cycle continues till the whole hubs in the network are clustered. The auxiliary cluster nodes are not the final cluster nodes. The clustering process predicts the best cluster nodes with a decrease in the cost function. The sensor nodes are arbitrarily initiated as fireflies before the selection of CHs. It is pretended that every firefly decides the opportunity of nodes being chosen as $\mathrm{CH}$ from the adjacent nodes. The attractiveness level of fireflies' $(0)$ is worthlessly decisive dependent on the Rand function. $\mathrm{CH}$ is elected based on the rank of nodes that has the most extreme number of neighboring nodes. The adjacent nodes send back joining messages to form the cluster. At that point, the $\mathrm{CH}$ node sends an acknowledgment to provide membership. Once the sensor nodes receive messages, it determines the Euclidian distance to every $\mathrm{CH}$ and connects with the closer one. Finally, $\mathrm{CH}$ forwards it to the sink node. The attractiveness and light intensity of each firefly is compared with other fireflies to find the fitness value.

2) Attractiveness and Light Intensity: The power of light differs contrarily with the square of separation and the predefined absorption of $\mathrm{r}$ indicated as $I(r)$, where $\mathrm{r}$ is the distance from the source and it is identified by Equation (4).

$$
I(r)=I_{0} / r^{2}
$$

The power of light at source is $I_{0}$. On the other hand, the medium is given by the force of light, at that point the intensity is provided in Equation (5).

$$
I(r)=I_{0} \exp (\gamma r)
$$

Here, $\gamma$ is the retention coefficient referenced in Gaussian coefficient as Equation (6).

$$
I(r)=I_{0} \exp \left(\gamma r^{2}\right)
$$

If the intensity maximizes in the neighboring firefly the respective attractiveness of the firefly also improves. The attractiveness is mentioned as $\beta$ in Equation (7).

$$
\beta=\beta_{0} \exp \left(-\gamma r^{2}\right)
$$

where $\beta_{0}$ denotes the attractiveness function at $\mathrm{r}=0$. The gap among the two fireflies $y_{i}$ and $y_{j}$ is $r_{(i, j)}$ estimated as in Equation (8)

$$
r_{(i, j)}=\sqrt{\sum_{k=1}^{d}\left(y_{(i, k)}-y_{(j, k)}\right)^{2}}
$$

where $y_{(i, k)}$ implies the $k_{t h}$ component of the $i_{t h}$ firefly at a spatial coordinate $y_{i}$.

3) Movement: The attractiveness quality describes the development of firefly towards more attractive capacity and it is expressed in Equation (9).

$$
y_{i}=y_{i}+\beta_{0} e^{-\sigma r_{(i, j)}^{2}}\left(y_{j}-y_{i}\right)+\alpha
$$

where $\alpha$ indicates the randomization variable and $€$ denotes the attraction. The pseudocode for the firefly algorithm is mentioned in Algorithm 1. Figure 6 shows the flow chart of the firefly algorithm for clustering.

\section{A. Cluster Head Formation}

Cluster head selection is an important phase in the network for balancing energy between clusters. In general, this phase is processed by auxiliary cluster heads in the cluster. The ideal cluster head is selected dependent on the best qualities like high residual energy, more number of neighbor hubs, and the shortest distance from the base station. The network consumes minimum energy during data transmission. At any point, if the $\mathrm{CH}$ faces any problem due to the battery discharge, then there will be no possible ways to find another cluster head in the network. The $\mathrm{CH}$ is formed on the basics of the quantity of energy left behind the nodes; the nodes with higher energy will be a cluster head. Therefore, ANN is used in this paper to find the maximum energy values among all the nodes to find the best $\mathrm{CH}$. 
Input: Maximum iterations, Dimensions, population, light absorption coefficient $(\lambda)$, Attraction coefficient $\left(\beta_{0}\right)$, mutation coefficient $(\alpha)$, Uniform mutation range (delta)

Output:Clustered nodes

Initialize population

For $\mathrm{i}=1$ : maximum iterations

Fitness evaluation

Distance calculation between neighbouring nodes

Calculation of attractiveness for neighbouring fireflies

For $\mathrm{j}=1$ : number of fireflies

For $\mathrm{k}=1$ : number of fireflies

$$
\text { If } I_{j}<I_{k}
$$
end

Move firefly $\mathrm{i}$ towards firefly $\mathrm{j}$

Update new firefly position end

end

Rank and find best fireflies

end

Cluster creation

Algorithm 1: Pseudocode for firefly clustering algorithm

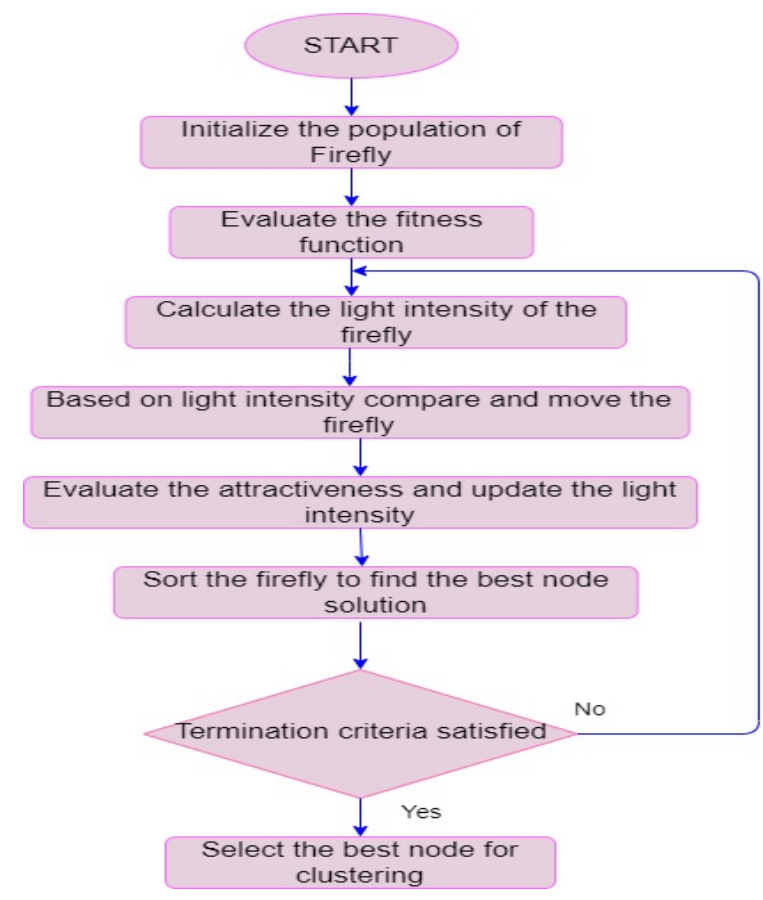

Fig. 6. Clustering using Firefly Algorithm.

\section{B. Chain Formation}

Generally, the energy consumption will be less in chainbased transmission. Hence, PEGASIS double cluster head formation is implemented to pass data to the sink node. PDCH maintains the capacity level of every node to improve the network lifetime. The proposed method makes use of two $\mathrm{CHs}$ as shown in Figure 7 rather than one in a single chain. The main purpose of selecting two $\mathrm{CHs}$ is to avoid the formation of a long chain in the network. PDCH exceeds the function of PEGASIS in terms of disposal of cluster overhead formation, limiting the distance between nodes, minimizing the transmissions between nodes, and allowing only single transmission for the base station in one round.

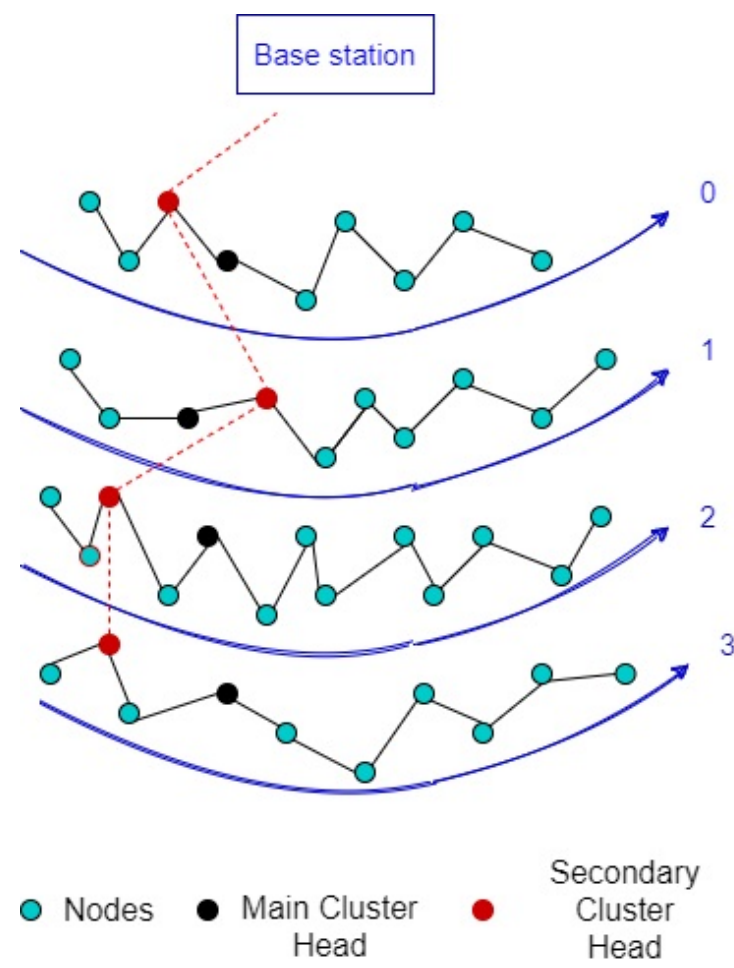

Fig. 7. Double cluster head chain.

\section{Secondary Cluster Head Selection}

The chain formed within the primary heads is known as the main chain and the head in the main chain is considered as secondary cluster head. The secondary cluster head is estimated about the gap between the primary head from the base station. The primary cluster head closer to the base station is elected as the secondary cluster head. The main characteristic of the secondary cluster head is to sense information from the primary cluster head and transfer it to the base station. The data transmission to the base station will diminish some energy in the network during this process. The $\mathrm{SCH}$ rescues the energy utilization of cluster heads which is aside from the base station. If a chain selects a dual $\mathrm{CH}$ for data transfer, then it is necessary to assign a time for the transmission of data. The throughput decreases due to this process. The primary aim of this algorithm is to minimize energy utilization between the hubs and the base station. There is a point present at the chain between the $\mathrm{CH}$ and the base station called the extraordinary point utilized for electing the SCHs. This proves that less energy is used for transferring packets to the base station. If the energy of the node is greater than the threshold value, then the nodes are alive and data is communicated to the BS. The pseudocode for GWO is illustrated in Algorithm 2. 


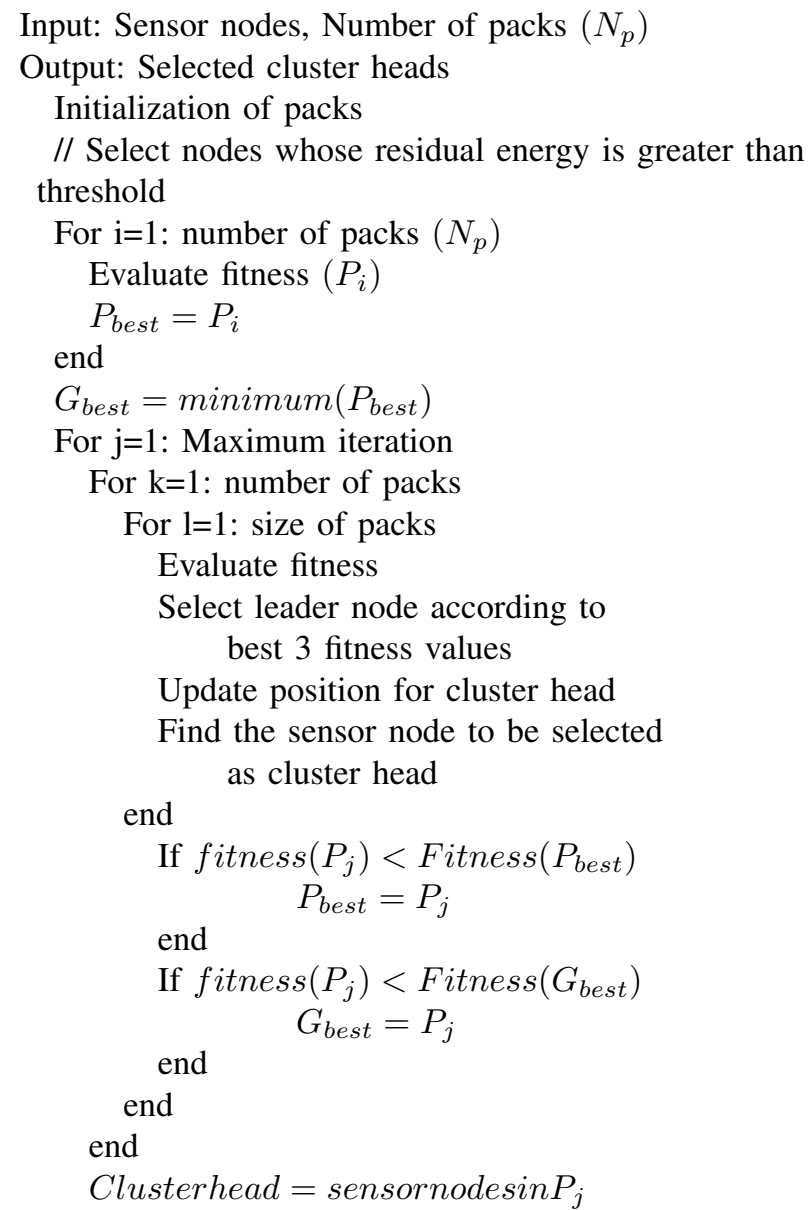

Algorithm 2: Pseudocode for GWO based secondary cluster head selection

During hunting, the prey is surrounded by the grey wolves whose position is determined as given in Equation (10). The next position of the wolf is found by Equation (11).

$$
\begin{gathered}
\vec{F}=\vec{D}\left(\vec{S}_{v(x)}(t)-\vec{S}\right) \\
\vec{S}(t+1)=\vec{S}_{v(x)}(t)-\vec{F} \vec{B}
\end{gathered}
$$

where $\mathrm{t}$ indicates the number of iteration and $\vec{S}_{v(x)}(t)$ is the vector position of prey and $\vec{S}, \vec{F}$ is the vector position of grey wolf. $\vec{B}$ and $\vec{D}$ are the coefficient matrix calculated using Equations (12) and (13).

$$
\begin{gathered}
\vec{D}=2 \vec{v}_{y} \vec{r}_{p}-\vec{v}_{y} \\
\vec{B}=2 \vec{r}_{q}
\end{gathered}
$$

Here, $\vec{r}_{p}$ and $\vec{r}_{q}$ are considered as a random vector between the range of 0 to 1 . Where, $\vec{r}_{p}$ and $\vec{r}_{q}$ vectors play an important part in assembling towards an optimal chain selection. Further, $\vec{v}_{y}$ is the control vector.The fitness function is $\alpha, \beta$ and $\delta$, and the total distance is denoted $\vec{D}$. The mathematical design of hunting characteristics of the grey wolves surrounding the prey is derived from Equations (10) and (11) for $\alpha, \beta$ and $\delta$ wolves as illustrated in Equations (14) $-(19)$.

$$
\begin{aligned}
& \vec{F}_{\alpha}=\left|\vec{C}_{1} \vec{S}_{\alpha}-\vec{S}\right| \\
& \vec{F}_{\beta}=\left|\vec{C}_{2} \vec{S}_{\beta}-\vec{S}\right| \\
& \vec{F}_{\delta}=\left|\vec{C}_{3} \vec{S}_{\delta}-\vec{S}\right| \\
& \vec{S}_{1}=\vec{S}_{\alpha}-\vec{D}_{1}\left(\vec{Z}_{\alpha}\right) \\
& \vec{S}_{2}=\vec{S}_{\beta}-\vec{D}_{2}\left(\vec{Z}_{\beta}\right) \\
& \vec{S}_{3}=\vec{S}_{\delta}-\vec{D}_{3}\left(\vec{Z}_{\delta}\right)
\end{aligned}
$$

The wolves should follow the alpha, beta and delta wolves to estimate the social structure. Since the position of the prey is not known, the $\alpha, \beta$ and $\delta$ wolves are considered the best solution because of knowing the position of the prey. Hence, it is demonstrated that the position of the prey is estimated by updating the positions of alpha, beta and delta by following the Equations (14) - (19). Finally, the modernized location of the wolf is obtained by Equation (20) expressed below.

$$
\vec{S}(t+1)=\vec{S}_{1}+\vec{S}_{2}+\vec{S}_{3} / 3
$$

The quest for prey begins when the rest of the wolves separate one another to discover the prey. This search is additionally subjected to the places of alpha, beta, and delta individuals. The flow chart of GWO for selecting the secondary cluster head is shown in Figure 8.

1) Computation of the fitness function: The distance between the sensor nodes is calculated for the position of the cluster head. The sensor nodes consuming some energy while transmission of data to the respective cluster head is represented in Equation (21). Where, $\mathrm{S}$ is the collection of all sensor nodes and $\mathrm{C}$ denotes the collection of all $\mathrm{CH}$.

$$
\vec{F}_{1}=\sum_{i=1}^{m}\left(\frac{1}{l i} \Sigma_{k=1}^{l i} \operatorname{dis}\left(s_{k}, C H_{i}\right)\right)
$$

where $F_{1}$ is the average intracluster distance, $\mathrm{m}$ is the total number of $\mathrm{CHs}$, li indicates the number of sensor nodes in the cluster $i, s_{k}$ defines the communication range of nodes, dis $\left(s_{k}, \mathrm{CH}_{i}\right)$ represents the distance between the sensor nodes $s_{k}$ and the cluster head $\mathrm{CH}_{i}$. The average distance of the sink $F_{2}$ is calculated by Equation (22) based on the ratio of separation among the cluster head and the base station to the whole sensor nodes present in the $\mathrm{CH}$. Hence, distance reduction is the basic logic to minimize the energy consumption.

$$
\vec{F}_{2}=\sum_{i=1}^{m}\left(\frac{1}{l i} \Sigma_{k=1}^{l i} \operatorname{dis}\left(C H_{i}, B S\right)\right)
$$

where $d s\left(C H_{i}, B S\right)$ denotes the distance between cluster head $\mathrm{CH}_{i}$ and Base station (BS). Moreover, the lifetime probability 
of the network relies upon the usage of energy where $F_{3}$ indicates the residual energy expressed in Equation (23).

$$
\vec{F}_{3}=\frac{1}{\sum_{i=1}\left(E_{C H i}\right)}
$$

Due to the random clustering process, there will be some small and large cluster formations. Thus, there is a need to balance the cluster. Where, $E_{C} H i$ represents the energy of current $\mathrm{CH}(\mathrm{CHi}) . \mathrm{F}_{4}$ is the $\mathrm{CH}$ balancing factor obtained by Equation (24).

$$
\vec{F}_{4}=\Sigma_{i=1} \frac{a}{b}-l i
$$

The variable a denotes the number of alive sensor nodes and $b$ indicates number of $\mathrm{CHs}$. To reduce all the fitness functions, it is better to minimize the combination of the above-mentioned fitness as in Equation (25).

Fittnessfunction $=p * d_{1}+q * d_{2}+r * d_{3}+(1-p+q+r) * d_{4}$

$\mathrm{p}, \mathrm{q}, \mathrm{r}$ is a constant value and $p+q+r=1$

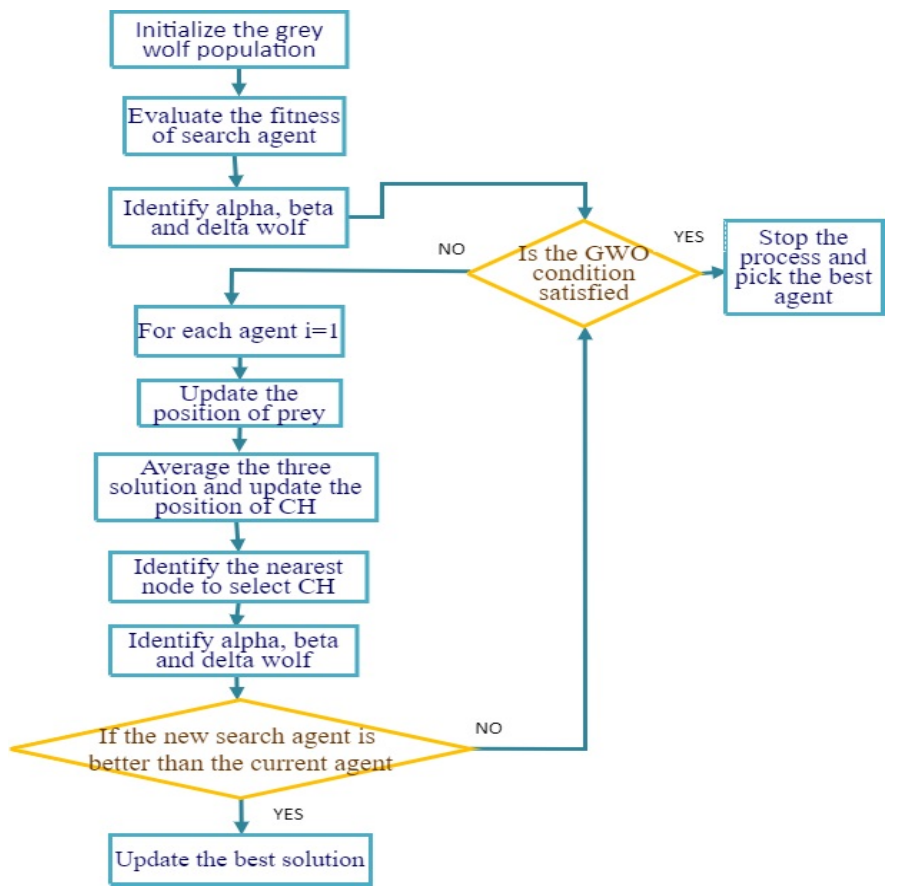

Fig. 8. Secondary Cluster Head selection by GWO.

\section{EXPERIMENTAL RESUlTS AND ANALYSIS}

The MATLAB R2018a simulator is used to analyze the proposed PDCH-ANN algorithm and predict the results for the experiment. The simulation parameters are listed in Table 1. Here, we analyze our method with LEACH and PDCH. The results of the mentioned techniques are compared based on some quality parameters like the number of alive nodes, average energy consumption, packet delivery ratio with energy efficiency, and throughput. Moreover, the proposed method has been experimented and the results are obtained. The simulation area is determined as $250 \times 250$ with 100 sensor nodes in a fixed sink node. The analysis of the implemented method is explained in this section.

TABLE I

Simulation PARAMETERS

\begin{tabular}{|c|c|}
\hline Parameter & Value \\
\hline Deployment of nodes & Random \\
\hline Sink node & Fixed \\
\hline Number of nodes & 100 \\
\hline Network area & $250 \times 250$ \\
\hline Size of data packets & 1024 \\
\hline Initial energy of nodes & $0.5 \mathrm{~J}$ \\
\hline Circuit energy consumption per bit & $50 \mathrm{~nJ} / \mathrm{bit}$ \\
\hline Transmits energy & $50 \mathrm{~nJ} / \mathrm{bit}$ \\
\hline Receive energy & $50 \mathrm{~nJ} / \mathrm{bit}$ \\
\hline $\begin{array}{l}\text { Energy spent by amplifier to } \\
\text { transmit bits for large distance }\end{array}$ & $0.0013 \mathrm{pJ} / \mathrm{bit} / \mathrm{m} 4$ \\
\hline $\begin{array}{l}\text { Energy spent by amplifier to } \\
\text { transmit bits for small distance }\end{array}$ & $10^{3} \mathrm{pJ} / \mathrm{bit} / \mathrm{m} 2$ \\
\hline Data aggregation energy & $5 \mathrm{~nJ} / \mathrm{bit} / \mathrm{signal}$ \\
\hline
\end{tabular}

\section{A. Lifetime of the Network}

The comparison of the first, half and last node dead is shown in Figure 9. The lifespan of the network is illustrated for certain rounds of first, half, and last node dead. The protocol used in our method is a cluster-based routing protocol that attains maximum lifetime when compared to other techniques like LEACH and PDCH. The lifespan of the network is predicted by the starting time of network setup to the time of completion over certain rounds. This estimates the number of alive nodes in the network with the number of first, half, and last node dead values as in Table 2. It is analyzed that the proposed method has more lifetime than the existing methods over certain rounds. It is achieved due to the selection of $\mathrm{CH}$ via ANN that minimizes the cluster distance.

TABLE II

COMPARISON WITH EXISTING TECHNIQUES

\begin{tabular}{|l|l|l|l|l|}
\hline Techniques & $\begin{array}{l}\text { First node } \\
\text { dead }\end{array}$ & $\begin{array}{l}\text { Half node } \\
\text { dead }\end{array}$ & $\begin{array}{l}\text { Last node } \\
\text { dead }\end{array}$ \\
\hline Proposed & 612 & 1094 & 1276 & \\
\hline PDCH & 257 & 621 & 1083 & \\
\hline LEACH & 121 & 633 & 1001 & \\
\hline
\end{tabular}

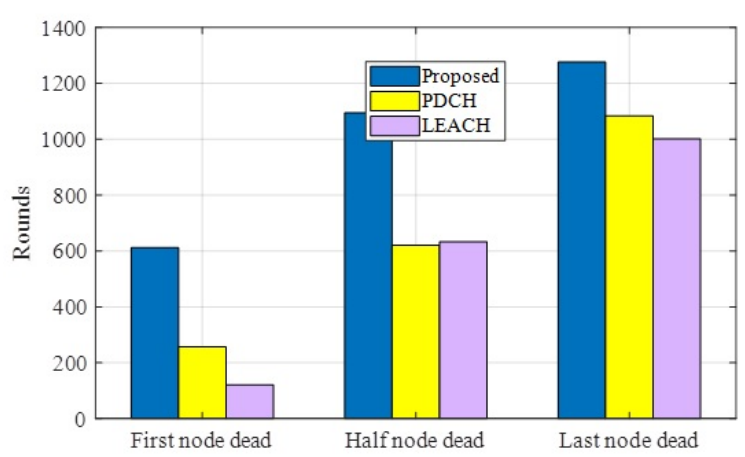

Fig. 9. Comparison of methods with First, Half and Last node dead 


\section{B. Number of Alive Nodes}

This section explains the rate of routing protocol in determining the number of alive nodes in the network in connection with the other two techniques as shown in Figure 10. All the methods including the proposed technique sustain a greater number of alive nodes. The proposed method has recorded the highest number, the PDCH is the second and the LEACH is the last. This happens because of the selection of cluster heads among the clusters through ANN. ANN selects the node with high residual energy and a greater number of adjacent nodes.

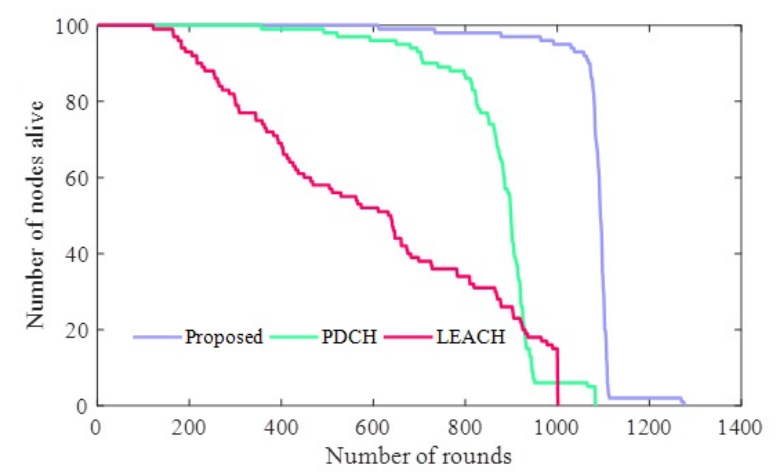

Fig. 10. Rounds (VS) Number of alive nodes

\section{Average Energy Consumption}

The average energy consumed in the proposed technique is related to the existing methods such as LEACH and PDCH as shown in Figure 11. There are two illustrations concerning the nodes and rounds. The energy consumed by the proposed method is minimum where the other two methods consume more energy to the energy level of nodes. PEGASIS possesses a low energy consumption protocol with the chain formation in the network. The average energy consumed per round also gets minimized when compared to the other methods. Finally, the proposed method addresses the problem of unstable network traffic by assuming a congestion gradient in transferring data to the base station with minimum consumption of energy.

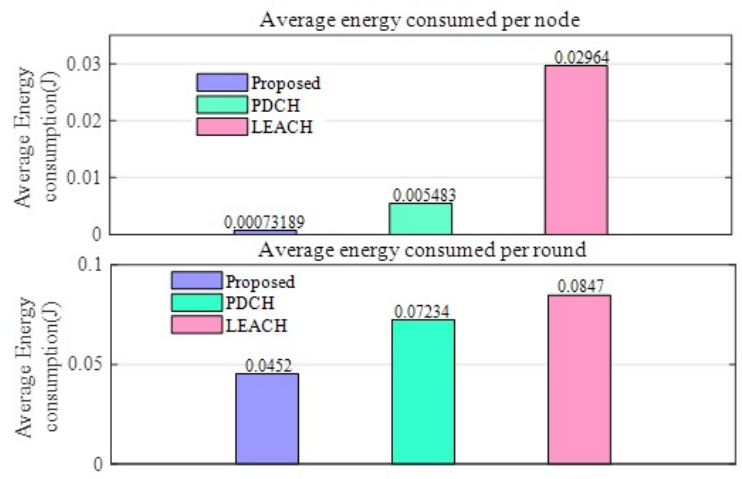

Fig. 11. Average energy consumption with number of nodes \& rounds

\section{Number of Packets Sent}

The results of the number of packets transferred to the sink node in comparison with LEACH and PDCH are shown in Figure 12. The total number of packets send to the BS is 12,547 and to the $\mathrm{CH}$ are 46839 as mentioned in Table 3. This has been achieved due to the number of divided clusters in the network. The proposed algorithm selects perfect $\mathrm{CH}$ (primary and secondary $\mathrm{CH}$ ) in the network. If a greater number of packets are sent, then network efficiency also gets maximized with an increase in network throughput.

TABLE III

NUMBER OF PACKETS SEND TO BS AND CH

\begin{tabular}{|l|l|l|l|}
\hline & Proposed & PDCH & LEECH \\
\hline $\begin{array}{l}\text { Packets send } \\
\text { to BS }\end{array}$ & 12,5487 & 111009 & 80,858 \\
\hline $\begin{array}{l}\text { Packets send } \\
\text { to CH }\end{array}$ & 46839 & 27234 & 15478 \\
\hline
\end{tabular}

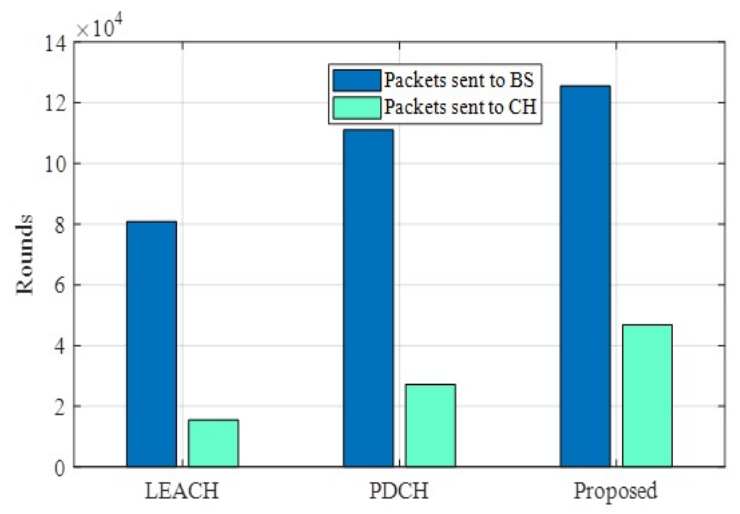

Fig. 12. Total number of packets send to $\mathrm{BS}$ and $\mathrm{CH}$

\section{E. Packet Delivery Ratio}

The packet delivery ratio (PDR) of the proposed protocol is evaluated using various numbers of nodes and compared with LEACH and PDCH as shown in Figure 13. It is absorbed that if the number of the node gets an increase, the delivery ratio also gets higher due to the estimation of $\mathrm{CH}$ in the proposed cluster-based routing protocol. The main logic behind this is $\mathrm{CH}$ selecting the neighboring nodes for information transmission dependent on the remaining energy and queue length. Table 4 displays the values of the packet delivery ratio. The PDR is higher than the conventional methods due to the successful transfer of data to the sink node. Moreover, the latency in PDR is diminished due to the uniform circulation of traffic and energy against the BS via firefly-based clustering protocol.

\section{F. End to End Delay}

The end-to-end delay comparison of the proposed strategy is shown in Figure 14. The delay metric in the network must be designed efficiently because the delay arrival of packets in real-time applications can cause a wide effect. For instance, a minor delay in the healthcare monitoring of 
TABLE IV

PACKET DELIVERY RATIO

\begin{tabular}{|l|l|l|l|}
\hline & Nodes $=50$ & Nodes $=75$ & Nodes $=100$ \\
\hline Proposed & 70 & 79 & 92 \\
\hline PDCH & 63 & 70 & 87 \\
\hline LEECH & 60 & 72 & 85 \\
\hline
\end{tabular}

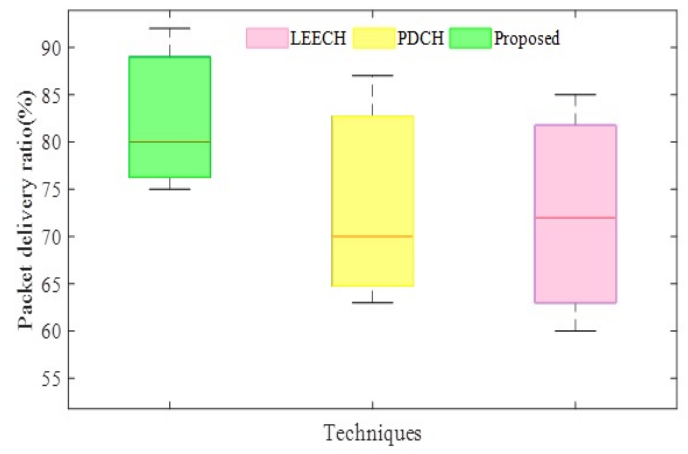

Fig. 13. Packet delivery ratio with number of nodes

TABLE V

END TO END DELAY COMPARISON

\begin{tabular}{|l|l|l|l|}
\hline & Nodes $=50$ & Nodes $=75$ & Nodes $=100$ \\
\hline Proposed & 2.589 & 5.4105 & 7.1264 \\
\hline PDCH & 4.1414 & 7.0354 & 12.654 \\
\hline LEECH & 10.258 & 15.483 & 21.987 \\
\hline
\end{tabular}

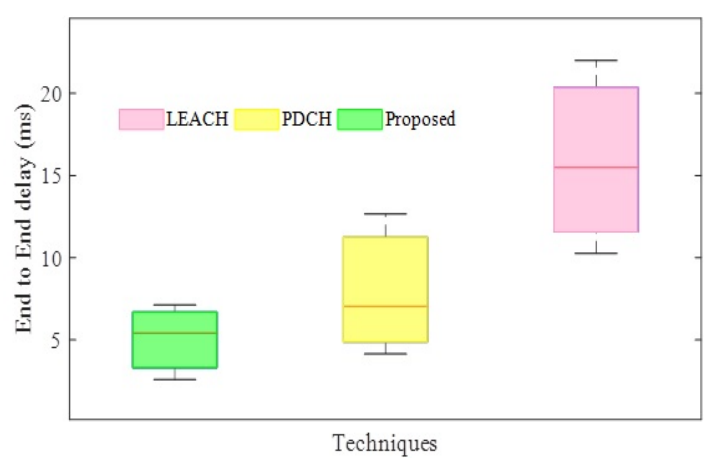

Fig. 14. End to End Delay comparison with other methods

the patients in the medical industry can cause a risk to the patient's life. Hence, the delay parameter should be maintained accordingly. In general, end-to-end delay is characterized as the time required for transmitting packets from source to sink node in the network and the values are tabulated in Table 5. The proposed method has minimum delay compared to the existing methods. During routing between cluster heads, the path having minimum traffic is chosen for data transmission to decrease the delay in the network. The nodes with more energy are preferred to establish a path between BS and $\mathrm{CH}$. However, the expansion in the number of network nodes prompts more extended high end-to-end delay since congestion brings more packet drop.

\section{G. Throughput and Energy Efficiency}

The performance of energy efficiency and throughput is shown in Figure 15. It is imperative to design the protocols to tackle congestion in an efficient manner, to reduce packet loss, and to increase the network lifetime. The cluster-based routing protocol is proposed to improve all these parameters. The throughput and energy efficiency of the proposed network outperforms the existing methods where throughput is a measure of successful delivery of packets to the BS in a particular time interval. The proposed method has obtained $87.17 \%$ of throughput as tabulated in Table 6. This happens after selecting the $\mathrm{CH}$. It finds the best possible way to transfer the data to the base station by the proposed cluster-based routing protocol. Energy efficiency in the network gets improved because of the selection of $\mathrm{CH}$ with ANN. Moreover, the nodes nearer to BS will get selected first, further $\mathrm{CH}$ and cluster member consumes minimum energy for data transmission thereby energy efficiency is increased.

TABLE VI

ENERGY EFFICIENCY \& THROUGHPUT

\begin{tabular}{|l|l|l|l|}
\hline & Proposed & PDCH & LEECH \\
\hline Throughput & 87.17 & 85.21 & 75.43 \\
\hline $\begin{array}{l}\text { Energy effi- } \\
\text { ciency }\end{array}$ & 93.33 & 82.97 & 75.67 \\
\hline
\end{tabular}

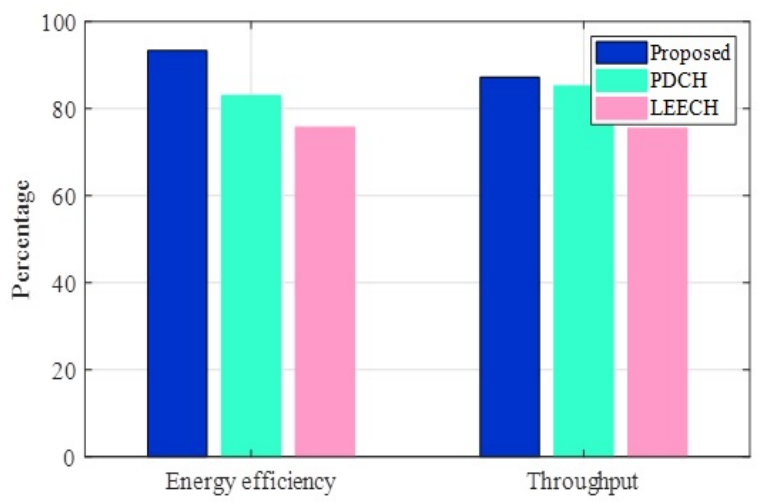

Fig. 15. Performance of Energy Efficiency and Throughput

\section{CONCLUSION}

In this paper, the proposed method utilizes PEGASIS Double cluster head with ANN to analyze the overall network lifetime. The existing challenges in WSNs are congestion control as an increase in congestion leads to throughput reduction, energy loss, decrease in energy efficiency, and packet loss. Therefore, a hybrid method is proposed to reduce congestion by the cluster-based routing protocol. The proposed method has four phases: In the first phase, the sensor nodes are clustered via the Firefly algorithm. In the second phase, the cluster head node selection is performed with ANN, and chain formation is processed through PEGASIS double cluster head. In the fourth phase, the $\mathrm{SCH}$ is selected with GWO and the equivalence of energy consumed is predicted by the sensor hubs. Experimental results predict that the 
proposed method improves network lifetime when related to LEACH and PDCH techniques. Moreover, it increases the average energy consumption, packet delivery ratio, end-to-end delay, throughput, and energy efficiency of the network. The comparative analysis of the proposed method gains up to $87 \%$ of throughput and $93 \%$ of energy efficiency in the network. In future work, further routing schemes can be enhanced with the greater number of sensor nodes for IoT applications. Moreover, the routing protocol can be explained in detail with hierarchical clustering in a multi-hop method to improve the clustering quality.

\section{REFERENCES}

[1] P. Aimtongkham, T. G. Nguyen and C. So-In, Congestion Control and Prediction Schemes Using Fuzzy Logic System with Adaptive Membership Function in Wireless Sensor Networks, Hindawi Wireless Communications and Mobile Computing, 1-19, 2018. 10.1155/2018/6421717

[2] T. Bokareva, W. Hu, and S. Kanhere, Wireless Sensor Networks for Battlefield Surveillance, 2006.

[3] H. Alemdar and C. Ersoy,Wireless sensor networks for healthcare: a survey. Computer Networks, 54(15), 2688-2710, 2010. 10.1016/j.comnet.2010.05.003

[4] H. K. Patil and M. C. Thomas, Wireless Sensor Network Security in Computer and Information Security Handbook, (Third Edition), 2017

[5] S. L. Yadav and R. L. Ujjwal, Sensor data fusion and clustering: A congestion detection and avoidance approach in wireless sensor networks, Journal of Information \& Optimization Sciences, 2020. 10.1080/02522667.2020.1799512

[6] A. Sarkar and S. T. Murugan, Routing protocols for wireless sensor networks: What the literature says?, Alexandria Engineering Journal, 55(4), 3173-3183, 2016. 10.1016/j.aej.2016.08.003

[7] A. Hosen and G. Cho, An Energy Centric Cluster-Based Routing Protocol for Wireless Sensor Networks. Sensors, 18 (5), 1520,2018 $10.3390 / \mathrm{s} 18051520$

[8] N. Moussa, H. Z. Alaoui, E. Belrhiti and E. A. Alaoui, ECRP: an energy-aware cluster-based routing protocol for wireless sensor networks, Wireless Networks, Springer Science and Business Media, LLC, part of Springer Nature 2020, 2020.

[9] D. Sharma and A. P. Bhondekar, Traffic and energy aware routing for heterogeneous wireless sensor networks, IEEE Communication. Lett, 22,1608-1611, 2018. 10.1109/lcomm.2018.2841911

[10] T. Kaur and D. Kumar, Particle swarm optimization-based unequal and fault tolerant clustering protocol for wireless sensor networks, IEEE Sens. J,18, 4614-4622, 2018. 10.1109/jsen.2018.2828099

[11] T. M. Behera, U. C. Samal and S. K. Mohapatra, Energy-efficient modified LEACH protocol for IoT application, IET Wireless. Sens. Syst, 8, 223-228, 2018. 10.1049/iet-wss.2017.0099

[12] M. Baskaran and C. Sadagopan,Synchronous Firefly Algorithm for Cluster Head Selection in WSN, The Scientific World Journal, 1-7, 2015. $10.1155 / 2015 / 780879$

[13] V. Srivastava, S. Tripathi, K. Singh, and L. H. Son, Energy efficient optimized rate-based congestion control routing in wireless sensor network, Journal of Ambient Intelligence and Humanized, 2019. 10.1007/s12652019-01449-1

[14] M. Farsi, M. Y. Badaw, M. Moustafa, H. A. Ali and Y. Abdulazeem, A Congestion-Aware Clustering and Routing (CCR) Protocol for Mitigating Congestion in WSN, 2019 IEEE, 7, 2169-3536. Y, 2019. 10.1109/access.2019.2932951

[15] D. K. Sharma, D. Kukreja, S. Bagga and R. Rastogi,Gauss-sigmoid based clustering routing protocol for wireless sensor networks, International Journal of Information Technology, 2019. 10.1007/s41870-01900391-x

[16] A. S. Kirsan, U. A. Harun, M. Rasyid and I. Syarif, Efficient Energy for Cluster Head Selection using New LEACH-based routing protocol in Wireless Sensor Network 2019 International, 2019. 10.1109/elecsym.2019.8901669

[17] P. Gupta and A. K. Sharma, Clustering-based heterogeneous optimized-HEED protocols for WSNs, Soft Computing,1737-1761, 2019. 10.1007/s00500-017-2837-7

[18] S. H. Sackey, J. A. Ansere, J. H. Anajemba, M. Kamal and C. Iwendi, Energy Efficient Clustering Based Routing Technique in WSN using Brainstorm Optimization, 2019 15th International Conference on Emerging Technologies (ICET), 2019. 10.1109/icet48972.2019.8994740
[19] M. S. Tomar and P. K. Shukla, Energy Efficient Gravitational Search Algorithm and Fuzzy Based Clustering with Hop Count Based Routing for Wireless Sensor Network, Multimedia Tools and Applications, 2019. 10.1007/s11042-019-07844-2

[20] A. Hamzah, M. Shurman, O. Al-Jarrah and E. Taqieddin, EnergyEfficient Fuzzy-Logic-Based Clustering Technique for Hierarchical Routing Protocols in Wireless Sensor Networks, Sensors,19(3), 2019. 10.3390/s19030561

[21] S. Wang,B. Zhao, D. Li, and T. Du, Data Fusion Algorithm of Wireless Sensor Based on Combination between Cluster Head Election Improvement and Neural Networ, 2019 Chinese Control, 2019. 10.23919/chicc.2019.8866153

[22] J. Wang, Y. Gao, X. Yin, F. Li and H. J. Kim, An Enhanced PEGASIS Algorithm with Mobile Sink Support for Wireless Sensor Networks, Hindawi Wireless Communications and Mobile Computing, 2018. 10.1155/2018/9472075

[23] T. Li, F. Ruan, Z. Fan, J. Wang and J. U. Kim, An Improved PEGASIS Routing Protocol Based on Neural Network and Ant Colony Algorithm, International Journal of Future Generation Communication and Networking, 8 (6), 149-160, 2015.

[24] S. L. Yadav and R. L. Ujjwal, Sensor data fusion and clustering: A congestion detection and avoidance approach in wireless sensor networks, Journal of Information and Optimization Sciences, 2020. 10.1080/02522667.2020.1799512

[25] X. S. Yang, Nature-Inspired Metaheuristic Algorithms, 2008.

[26] P. Goswami, Z. Yan, A. Mukherjee, L. Yang, S. Routray and G. Palai, An Energy Efficient Clustering using Firefly and HML for Optical Wireless Sensor Network, Optic, 2019. 10.1016/j.ijleo.2018.12.191

[27] S. Ali and R. Kumar, Artificial Intelligence Based Energy Efficient Grid PEGASIS Routing Protocol in WSN, 2018 7th International Conference on Reliability, Infocom Technologies and Optimization (Trends and Future Directions) (ICRITO), 2018. 10.1109/icrito.2018.8748501

[28] J. Rana, S. Vhatkar and M. Atique, Comparative Study of PEGASIS and PDCH Protocols in Wireless Sensor Network, International Journal of Computer Applications, 2015.

[29] D. Agrawal, W. M. H. Qureshi, P. Pincha, Srivastava, S. Agarwal, V. Tiwari and S. Pandey, GWO-C: Grey wolf optimizer-based clustering scheme for WSNs, International Journal of Communication Systems, e4344, 2020. 10.1002/dac.4344

[30] P. Subramanian, J. M. Sahayaraj, S. S. Kumar and D. S. Alex, A Hybrid Grey Wolf and Crow Search Optimization Algorithm-Based Optimal Cluster Head Selection Scheme for Wireless Sensor Networks, Wireless Personal Communications, 2020. 10.1007/s11277-020-07259-5

[31] S. M. M. H. Daneshvar, P. A. A. Mohajer and S. M. Mazinani, Energy-Efficient Routing in WSN: a Centralized Cluster-Based Approach via Grey Wolf Optimizer, IEEE Access, 1-1, 2019. 10.1109/access.2019.2955993

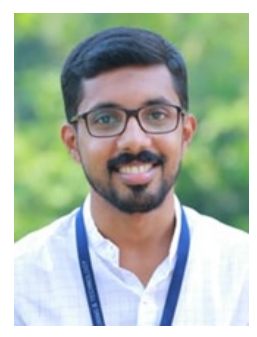

Abdul Ali is a Research Scholar in Computer Science and Engineering at Sathyabama Institute of Science and Technology, Chennai, India. He is having 11 years of experience in teaching. He received his B.Tech. Degree from M.G. University, Kerala, India. He received his M.Tech. Degree from M.S. University, Tirunelveli, India. He has published more than 10 papers in National and International Conference/Journals. His research interest includes Wireless Communication, Wireless Sensor Network.

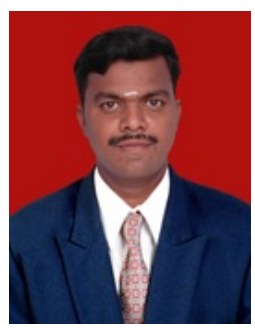

M.Vadivel is an Associate Professor in Electronics and Communication Engineering at Vidya Jyothi Institute of Technology, Hyderabad, Telangana, India. $\mathrm{He}$ is having 16 years of experience in teaching. He received his B.E. Degree from University of Madras, India. He received his M.E and Ph.D. degree from Sathyabama University, India. He has published more than 40 papers in National and International Conference/Journals. His research interest includes Wireless Communication, Wireless Sensor Network, Digital Image Processing and VLSI Design. 